\title{
Search for mathematical proof in the Web environment as a process of creative collective discovery
}

\author{
Ioannis M. Vandoulakis ${ }^{1, *}$ \\ ${ }^{1}$ The Hellenic Open University, School of Humanities. GR-15773 Zografou, Athens, Greece
}

\begin{abstract}
Although cooperation and collective efforts have always played an important role in the process of scientific discovery, recent technological innovations, such as the Internet, facilitate and encourage cooperation and collective effort to an unprecedented degree. This applies to all fields of science, even mathematics, where the process of mathematical discovery has traditionally been considered as an individual affair. This perspective requires new research on the theoretical level, and reveals the importance of various aspects of the process of scientific discovery in relation to the issues of problem solving, discovery and creativity, which have not been studied sufficiently.
\end{abstract}

\section{Proofs vs. proof events}

The use of the Internet encourages the participation in the search for a mathematical proof of people of different viewpoints, interests, educational background and expertise. This inevitably affects the practice of mathematical proving and a fortiori the concept of mathematical proof $[1,2]$.

Modern mathematical logic has developed powerful tools for the formal representation of proof in very different ways. Since mathematical proofs are represented in a formal language theory, proof theory confines itself only to those propositions, which can be expressed in such a formalism. This is the famous Hilbert's program [3]. However, the traditional approach to the concept of mathematical proof cannot capture the diversity of the process of mathematical proving, in particular proving in the Web environment. Thus, we adopt a new, extended definition of mathematical proof, introduced by Joseph Goguen (1941-2006) namely, the concept of proof events [4]. This concept incorporates not only the concluding outcome of the process of mathematical proving, but also the following aspects the process itself [5, 113-114; 6, 375]:

(1) Social aspect. Every proof event takes place in space and time with the participation of a definite community.

(2) Medium of communication. During a proof event, certain communication media are used, such as text (handwritten, printed, electronic, etc.) formulated in a (standard or formal) language or semiotic code, oral discourse (lectures, etc.), visual images (graphs, Javaapplets, etc.), as well as communication through practices.

(3) Prover vs. interpreter. A proof event presupposes two agents: a prover, who can be human or machine, or their combination, and an interpreter, who can be human, machine, or groups of them, or combination of them.

(4) Interpretation. This concerns the definition of meaning of the signs of the semiotic code that are used for the representation of a purported mathematical proof. During this process, an interpreter may change the initial proof, suggested by a prover, by adding new definitions, filling possible gaps, etc.

(5) Understanding and validation. A proof event is considered complete, whenever the participants involved in it conclude that they have understood the proof and that what has been suggested is actually a proof.

(6) History. Insofar as a proof event takes place in space and time, it has history; the codes of representation and communication of a proof is also embedded in history.

(7) Style. Proof events generate proofs formulated in various styles, characterizing different cultures, schools or individual scholars, who might share different views of mathematical rigor and other concepts of meta-theoretical character [5, 121-123; 7, 237-241].

The concept of proof event can be formalized in the calculus of events of Kowalski type [8,9]. Using the language of the calculus of events, we can talk about proof events and their sequences, evolving in time [10]. The semantics of proof events follow a logic that can be expressed in terms of Kolmogorov's calculus of problems [11], initially used for explication of intuitionistic logic $[12,10]$. 


\section{Specifics of the Web as a medium of mathematical proving}

The conception of proof events was used to describe the mathematical practice developed within the Kumo and Polymath projects, initiated by J. Goguen [13] and William Timothy Gowers [14], respectively [15]. The search for mathematical proof is in the Web is a new kind of practice, characterized by the change of the communication environment: The Web serves not only as a source of information, i.e. as a repository of information, ideas and methods, but also as the medium of communication, notably as a means to create global communities. In both Kumo and Polymath projects, parts of a proof can be exchanged among the members of a group and Web communication becomes an essential part of the proving activity. Nielsen [16] suggested the term "networked science" to describe this novel mathematical proving practice.

Some of the characteristics of this practice that deeply affect our views on mathematical proving are the following:

- The use of a new medium for transmission of information, notably the Web, which is characterized by openness and the capacity to form interest-centred communities.

- The interactivity of the Web tools that facilitates the exchange of ideas and methodologies and makes the participants in the proof event to behave as a goal-directed system of agents. This resulted in an unpredictable intensification of the proving process [17].

- The interactivity of the Web enables the use of brainstorming [18] as a means of investigation coordinated and ultimately integrated by the Web administrator. In the Polymath project, this method was assumed the form of (asynchronous) networked brainstorming [19] that made possible to take advantage of the complementarity of the mathematical skills of the participants. The level of competence that such a group can reach is not reducible to the sum-total of its constituents: it is not just the sum-total of knowledge of these people from different areas of mathematics.

- Web-based proving incorporates features of crowdsourcing [20] insofar as the search process for a proof in the Web is initiated by the administrator of the Web tool and proceeds in a collective manner. However, the final product, i.e. the ultimate proof, cannot be attributed to the initiator of the proof event. It represents a collective intellectual property with identifiable individual contribution by every participant.

\section{Modelling Web-based proof events in terms of cognitive architectures}

It has been shown [21] that Web-based proof events can be expressed in terms of codelets and cognitive architectures of Hofstadter type [22]. The aim of codelets is to facilitate the construction of conceptual structures in the operative memory, whenever a signal is inputted. They work in parallel, independently towards a specific goal. A codelet might generate a whole hierarchy of new codelets that work on sub-tasks of the initial problem $[21,41 ; 6,373]$.

A Web-based proof event can then described as follows. An agent (participant) involved in a proof event may be regarded as codelet. A problem can be posed by the supervising codelet, i.e. an agent of wide profile. The participants of the proof event can be any agent, who feels that possesses adequate abilities to solve the problem and is willing to spend time and energy to this effect. Further, an agent acting as a codelet can pose subtasks of simpler and narrower nature, which may be attacked by less skilled or less specialized agents, etc. In this way, a proof event of high complexity can be fulfilled by agents of lower capacities. Thus, a Webbased proof event can be modelled as a goal-directed cooperative activity of a system of codelets (community of agents) [21, 43; 6, 379-380]. The traditional image of isolated brilliant mathematical mind, able to deal with complex mathematical problems, is replaced in this case, by the image of "collective" mathematical mind, which is more efficient to do the same job in a shorter time.

\section{Application in mathematics education}

Proceeding from the analysis of the aforementioned case studies of Web-based proof events, we proposed a mental experiment for the study of collective problem solving and discovery. We described this discovery event in terms of cognitive architectures. The proof event starts with the statement of a task (problem): to prove the Pythagorean Theorem (PT) [6].

Thus, the initial state of the system is $\langle A, P T\rangle$, where $A$ is the supervising agent (codelet) of the proof event $P T$. The supervising agent can define the conditions (approaches) $P T_{i}(i=1,2,3,4)$ for the proving of $P T$. An agent $A_{i}$, who decided to take part in the proof event and undertake the role of a prover for one of the problems $P T_{i}$ starts acting (starts to search for a proof). Gradually, the system assumes a new state with four agents (codelets) $\left\langle A_{1}, P T_{1}\right\rangle,\left\langle A_{2}, P T_{2}\right\rangle,\left\langle A_{3}, P T_{3}\right\rangle,\left\langle A_{4}\right.$, $P T_{4}>$ that act as provers under the supervision of the agent (codelet) $A$. One agent (codelet), proceeding from his own mathematical culture and expertise, might develop a geometric approach towards the solution of the problem $P T$ and try to formulate an intuitive proof by conducting a "diagrammatic" style of reasoning. Another agent (codelet) who might be not satisfied or convinced by the intuitive proof or proceeding from his own background could approach the problem $P T$ algebraically and formulate an analytic proof; yet, another agent (codelet) can suggest a trigonometric proof, and so on.

In the course of the proof event, can be posed new sub-tasks (sub-problems), which generate subsequences of proof events, directed to the goal of solving the subproblems; this can take place with simultaneous enlargement of the system of agents (codelets). These 
sub-tasks may be the result of "noise" in communication, i.e. when certain points in the purported proof seem to be unintelligible or inconclusive, or may cause misunderstanding, because of the style used by the agent (codelet) that acts as prover, which might be not easily perceivable by another agent (codelet) acting as interpreter (See, for instance the historical episodes of reception of the ideas of Ramanujan, Lobachevsky and Brouwer, analysed in [23]).

The system stores all information about the history of development of a proof event (the problem solving process) and represents it in the form of co-operative non-linear discovery process that includes testing, possible confirmation or refutation of parts of a purported proof by the agents.

This model can be used in mathematics education, namely, in situations of cooperative problem solving in an environment of new information technologies (e.g. in a virtual environment). The most widespread today elearning systems (e.g. Moodle) enable collective problem solving in a virtual classroom of the platform (e-class). The role of administrator can be played by the teacher or by a student who has sufficient authority among his classmates. The role of agents can be played by the students, which can join a proof event at different times. Obviously, the agents (students) differ in capabilities; they may possess different levels of training and perception. Thus, they will handle a posed task very differently. They can undertake the role of a prover in their attempt to suggest an idea, argument or proof module, as well as the role of interpreter, whenever they attempt to understand, evaluate or dispute a purported proof, suggested by a prover agent (classmate). This switch of roles is not common in the traditional teaching methodologies, when before a student is posed a task (problem) and what is required is to fulfil the role of a proving agent to the ultimate proof of the problem (fulfilment of the task). However, learning is more effective, whenever a student is capable to switch roles and undertake the role of an interpreter who looks for possible gaps in a purported proof suggested by another agent (student) or attempts to evaluate an idea suggested by a classmate, i.e. whenever a student passes to the meta-cognitive level.

\section{References}

1. V.A. Bazhanov, Dokazatel'stvo. Trudy moskovskogo seminara po filosofii matematiki (URSS, Moscow, 2014) [In Rus]

2. I.M. Vandoulakis, P. Stephaneas, Conceptions of proof in mathematics, Dokazatel'stvo. Trudy moskovskogo seminara po filosofii matematiki (URSS, Moscow, 2014)

3. D. Hilbert, Gesammelte Abhandlungen, Vol. 3 (Springer, Berlin 1935).

4. J.A. Goguen, What is a proof. (2001). Available at URL:

http://cseweb.ucsd.edu/ goguen/papers/proof.html (accessed on March, 16, 2013)
5. P. Stefaneas, I. Vandoulakis, Philosophia Scientiæ, 18, 111-125 (2014)

6. P. Stefaneas, I. Vandoulakis, M. Martínez and H. Foundalis, In: T.R. Besold, M. Schorlemmer, A. Smaill (Eds) Computational Creativity Research: Towards Creative Machines (Atlantis, Springer, 2015)

7. I. Vandoulakis, P. Stefaneas, Mathematical Style as Expression of the Art of Proving, The $2^{\text {nd }}$ International Conference Science, Technology and Art Relations - STAR (With additional focus on Water, Energy and Space) In memory of Prof. Dror Sadeh, scientist and artist, 19-20 November 2014, Tel Aviv, Israel, (2014), 228-245. Available at URL:

http://engineers.org.il/_Uploads/12583STARS2-

BookofPapers.pdf. (accessed on March, 22, 2015)

8. R. Kowalski, S. Marek, New Generation Computing 4, 67-95 (1986)

9. R. Miller, M. Shanahan, Electronic Transactions on Artificial Intelligence, 3, 77-105 (1999)

10. P. Stefaneas, I. Vandoulakis, Journal of Artificial General Intelligence, 6, 130-149 (2015)

11. A.N. Kolmogorov, Selected Works of A.N. Kolmogorov. Vol. I: Mathematics and Mechanics (Kluwer, Dordrecht, 1991).

12. 2. I.M. Vandoulakis, P. Stephaneas, Trudy XII Mezhdunarodnykh Kolmogorovskikh Chtenii (Yaroslavl, 2014)

13. J.A. Goguen, Formal Aspects of Computing, 11, 272-301 (1999)

14. T.W. Gowers, Is massively collaborative mathematics possible? Available at Gowers' weblog URL: http://gowers.wordpress.com/2009/01/27/ismassively-collaborative-mathematics-possible/ (accessed on April, 13, 2015).

15. P. Stefaneas, I. Vandoulakis, Metaphilosophy. Special Issue: Philoweb: Toward a Philosophy of the Web: Guest Editors: Harry Halpin and Alexandre Monnin. Volume 43, Issue 4, 480-498, July 2012. Available at URL: http://web-andphilosophy.org. Reprinted in the collection: Harry Halpin and Alexandre Monnin (Eds) Toward a Philosophy of the Web. Wiley-Blackwell (2014), 149-167.

16. M. Nielsen, Reinventing Discovery: The New Era of Networked Science (Princeton University Press, 2011).

17. J. Rehmeyer, Science News. Available at URL: http://www.sciencenews.org/view/generic/id/50532/ title/Mathematics_by_collaboration, (accessed on: April 2, 2012).

18. A. Osborn, Applied Imagination: Principles and Procedures of Creative Problem Solving (Charles Scribner's Sons, New York, 1963).

19. A.R. Dennis, J.S. Valacich, Journal of Applied Psychology, 78, 531-537 (1993)

20. D.C. Brabham, The International Journal of Research into New Media Technologies, 14, 1, 7590 (2008)

21. P. Stefaneas, I. Vandoulakis, M. Martínez, H. Foundalis, "Web-based Mathematical Problem- 
Solving with Codelets" C3GI at ECAI 2012,

Computational Creativity, Concept Invention, and General Intelligence, 27 August 2012, Montpellier, France, (2012)

22. D.R. Hofstadter, Fluid Concepts and Creative Analogies: Computer Models of the Fundamental Mechanisms of Thought (Basic Books, New York 1995)

23. I. Vandoulakis, P. Stefaneas, Gaṇita Bhāratī, 35, 14, 119-157 (2013) 